\title{
Epigallocatechin gallate inhibits the growth of MDA-MB-231 breast cancer cells via inactivation of the $\beta$-catenin signaling pathway
}

\author{
ON-YU HONG ${ }^{1 *}$, EUN-MI NOH ${ }^{2 *}$, HYE-YEON JANG ${ }^{1}$, YOUNG-RAE LEE ${ }^{2}$, \\ BYOUNG KIL LEE ${ }^{3}$, SUNG HOO JUNG ${ }^{3}$, JONG-SUK KIM ${ }^{1}$ and HYUN JO YOUN ${ }^{3}$
}

\begin{abstract}
${ }^{1}$ Department of Biochemistry, Institute for Medical Sciences, Chonbuk National University Medical School, Jeonju, Chonbuk 54907; ${ }^{2}$ Department of Oral Biochemistry and Institute of Biomaterials Implant, School of Dentistry, Wonkwang University, Iksan, Chonbuk 54538 ; ${ }^{3}$ Department of Surgery, Research Institute of Clinical Medicine of Chonbuk National University, Biomedical Research Institute of Chonbuk National University Hospital, Jeonju, Chonbuk 54907, Republic of Korea
\end{abstract}

Received January 18, 2017; Accepted March 17, 2017

DOI: $10.3892 / \mathrm{ol} .2017 .6108$

\begin{abstract}
Epigallocatechin gallate (EGCG), a major constituent of green tea, has potential as a treatment for a variety of diseases, including cancer. EGCG induces apoptosis and inhibits tumorigenesis through multiple signaling pathways in breast cancer cells. $\beta$-catenin signaling modulators could be useful in the prevention and therapy of breast cancer. However, the precise anticancer effect of EGCG through the $\beta$-catenin signaling pathway in breast cancer is unclear. The present study investigated the association between $\beta$-catenin expression and clinicopathological factors of breast cancer patients, and the effect of EGCG on $\beta$-catenin expression in breast cancer cells. $\beta$-catenin expression was analyzed according to the clinicopathological factors of 74 patients with breast cancer. All patients were females diagnosed with invasive ductal carcinoma. Western blot analysis revealed that $\beta$-catenin was expressed at higher levels in breast cancer tissue than in normal tissue. $\beta$-catenin expression was associated with lymph node metastasis $(\mathrm{P}=0.04)$,
\end{abstract}

Correspondence to: Professor Hyun Jo Youn, Department of Surgery, Research Institute of Clinical Medicine of Chonbuk National University, Biomedical Research Institute of Chonbuk National University Hospital, 634-18 Geumam-dong, Deukjin, Jeonju, Chonbuk 54907, Republic of Korea

E-mail:yhj0903@jbnu.ac.kr

Professor Jong-Suk Kim, Department of Biochemistry, Institute for Medical Sciences, Chonbuk National University Medical School, 634-18 Geumam-dong, Deukjin, Jeonju, Chonbuk 54907, Republic of Korea

E-mail: jsukim@jbnu.ac.kr

${ }^{*}$ Contributed equally

Key words: epigallocatechin gallate, $\beta$-catenin, breast cancer, MDA-MB-231 cells tumor-node-metastasis stage $(\mathrm{P}=0.03)$ and estrogen receptor status $(\mathrm{P}<0.01)$. EGCG decreased MDA-MB-231 cell viability and significantly downregulated the expression of $\beta$-catenin, phosphorylated Akt and cyclin D1. Remarkably, additive effects of LY294002 and wortmannin, two phosphatidylinositol-3 kinase inhibitors, were observed. The present results suggest that EGCG inhibits the growth of MDA-MB-231 cells through the inactivation of the $\beta$-catenin signaling pathway. Based on these promising results, EGCG may be a potential treatment for triple negative breast cancer patients.

\section{Introduction}

Breast cancer is a highly heterogeneous disease, particularly in its locally advanced form (1). Despite the availability of various aggressive therapies for breast cancer patients, the mortality remains high (2). Thus, novel therapeutics are required for breast cancer patients. Numerous clinical and experimental studies have established that the clinical outcome of treatment for breast cancer depends on the expression of biological predictive markers such as estrogen receptor (ER) (3). Current effective prevention strategies for adjuvant therapy in ER-positive breast cancer include tamoxifen (4). However, ER-negative breast cancers are more clinically aggressive compared with ER-positive breast cancers, and their prognosis is poor due to the lack of hormone receptor (HR)-directed therapies $(1,4)$. Since there is no effective cure for ER-negative breast cancer patients, alternative interventions such as substances derived from natural herbal sources may be useful to replace the current regimens $(5,6)$. Therefore, new therapies or strategies are urgently required for patients with ER-negative and triple negative breast cancer (TNBC), which does not express any of the following three receptors: ER, progesterone receptor or human epidermal growth factor receptor 2 (HER2) (1).

The combination of polyphenols with a chemotherapeutic drug can result in additive or synergistic effects in ER-negative breast cancer cells (6). Furthermore, polyphenols induce restoration of tamoxifen sensitivity in TNBC cells (7). 
Green tea is one of the most widely consumed beverages in the world, and its ingestion in reasonable doses is considered safe (8). It has been reported that green tea consumption provides potential protection against numerous cancers through multiple mechanisms (8). Flavonoids in green tea are dietary factors that may protect against cancer, and have potent antioxidant effects (9). Epidemiological studies suggest that the low incidence of certain cancers in Asian countries is linked to the regular consumption of green tea $(9,10)$. There are reports linking green tea consumption with an improved prognosis in breast cancer $(11,12)$.

Epigallocatechin gallate (EGCG), a major polyphenol in green tea, has been extensively studied as a bioactive dietary agent against carcinogenesis, and it appears to act through multiple signaling pathways, including the mitogen-activated protein kinase, phosphatidylinositol-3 (PI3) kinase, epidermal growth factor receptor and nuclear factor- $\kappa \mathrm{B}$ signaling pathways (13-16). EGCG can inhibit breast tumorigenesis through the ER signaling pathway (17). In addition, EGCG enhances chemotherapeutic-induced cellular apoptosis in ER-negative MDA-MB-231 breast cancer cells (18), suggesting that EGCG exerts its anticancer properties through acting on ER signal transduction. However, the precise molecular mechanisms underlying this phenomenon are unclear.

Wnt signals are critical in regulating the normal development of the mammary gland, and dysregulation of Wnt signaling causes breast cancer (19). Wnt signaling cascades can be broadly subdivided into two categories: The canonical $\beta$-catenin signaling pathway and the non-canonical signaling pathway (19). Wnt/ $\beta$-catenin signaling is involved in several stages of growth and differentiation of the mammary gland, both during embryogenesis and following birth (19). This signaling system is pivotal in processes involved in the development and pathogenesis of breast cancer, including angiogenesis and hormonal signaling (20). EGCG induces the disruption of adherent junction formation and the accumulation of extra-nuclear $\beta$-catenin in MCF-7 cells $(19,20)$. The $\beta$-catenin signaling pathway is involved in EGCG-mediated anticancer protection (21). Therefore, members of the $\beta$-catenin pathway in tissues of breast cancer patients are potential targets of EGCG.

The present study investigated the anticancer effects of EGCG, the major active component of green tea (16), on MDA-MB 231 breast cancer cells. In addition, the expression profiles of the $\beta$-catenin signaling pathway in breast cancer patients were also analyzed. The results suggest that EGCG inactivates $\beta$-catenin signaling in MDA-MB 231 human breast cancer cells. Furthermore, $\beta$-catenin was significantly expressed at higher levels in ER-negative breast cancer patients compared with patients with ER-positive breast cancer. In summary, the present findings demonstrate that EGCG could be therapeutically effective in TNBC patients.

\section{Materials and methods}

Study subjects. The present study protocol was approved by the Institutional Review Board (IRB) of Chonbuk National University Hospital (Jeonju, Korea; IRB approval no. 2012-07-011). Among patients who underwent surgery at Chonbuk National University Hospital for primary breast cancers from June 2008 to July 2009, 74 breast cancer patients were identified and enrolled in the study. The patients' clinical and pathological characteristics were analyzed. The tumor-node-metastasis (TNM) stage was determined according to the 7th edition of the American Joint Committee on Cancer classification (22). Cancer and normal tissues (which were located away from the primary tumor site, histologically confirmed to be free of cancer cells and obtained from the same patient) were collected from all patients, who provided informed consent. The fresh tumor and normal background tissues were snap frozen in liquid nitrogen and stored at $-70^{\circ} \mathrm{C}$. Prior to immunoblotting, the tissue specimens were cut into small pieces and homogenized.

Cells and materials. MDA-MB-231 cells were obtained from the American Type Culture Collection (Manassas, VA, USA). Cells were cultured in high glucose-containing Dulbecco's modified Eagle's medium (DMEM) supplemented with $10 \%$ fetal bovine serum (FBS), 10,000 U/ml penicillin and $10,000 \mu \mathrm{g} / \mathrm{ml}$ streptomycin at $37^{\circ} \mathrm{C}$ in a $5 \% \mathrm{CO}_{2}$ incubator. EGCG was purchased from Sigma-Aldrich (Merck KGaA, Darmstadt, Germany). MTT and $\beta$-actin antibody (cat. no. A-5441) were obtained from Sigma-Aldrich (Merck $\mathrm{KGaA}$ ). The antibody against $\beta$-catenin (cat. no. Sc-59737) and horseradish peroxidase (HRP)-conjugated immunoglublin (Ig) G were purchased from Santa Cruz Biotechnology, Inc. (Dallas, TX, USA). The antibodies related to p-AKT (cat. no. 9271) and cyclin D1 (cat. no. 2922) were purchased from Cell Signaling Technology (Beverly, MA, USA). The PI3 kinase inhibitors LY294002 and wortmannin were purchased from Merck KGaA. High glucose-containing DMEM, FBS and PBS were obtained from Gibco (Thermo Fisher Scientific, Inc., Waltham, MA, USA).

Determination of cell viability. Cells were inoculated in a 96-well plate at a density of $3 \times 10^{4}$ cells/well, and incubated at $37^{\circ} \mathrm{C}$ for $24 \mathrm{~h}$ to allow attachment. The attached cells were either untreated or treated with EGCG for $24 \mathrm{~h}$ at $37^{\circ} \mathrm{C}$. Next, the cells were washed with PBS prior to the addition of MTT $(0.5 \mathrm{mg} / \mathrm{ml}$ in PBS$)$, and were incubated at $37^{\circ} \mathrm{C}$ for $30 \mathrm{~min}$. The formazan crystals were then dissolved with dimethyl sulfoxide (100 $\mu \mathrm{l} /$ well), and the absorbance was detected at $570 \mathrm{~nm}$ using a Model 3550 Microplate Reader (Bio-Rad Laboratories, Inc., Hercules, CA, USA).

Protein extraction. Breast cancer and adjacent normal tissues were collected immediately following surgery for protein extraction. These tissue samples were homogenized at $4^{\circ} \mathrm{C}$ in the presence of lysis buffer [50 mM Tris- $\mathrm{HCl}(\mathrm{pH} 8.0), 150 \mathrm{mM}$ $\mathrm{NaCl}, 5 \mathrm{mM}$ EDTA and 1\% NP-40]. The homogenates were subsequently centrifuged at $13,000 \mathrm{x}$ for $30 \mathrm{~min}$ at $4^{\circ} \mathrm{C}$, and the supernatants were collected. Upon washing with PBS, MDA-MB-231 cells were treated with EGCG, and then incubated for $24 \mathrm{~h}$ at $37^{\circ} \mathrm{C}$. These cells were lysed with ice-cold M-PER Mammalian Protein Extraction Reagent (Pierce; Thermo Fisher Scientific, Inc.). The protein concentration was determined using a DC Protein Assay kit (Bio-Rad Laboratories, Inc.).

Western blot analysis. The cell lysates (10 $\mu \mathrm{g}$ protein) were separated by $10 \%$ SDS-PAGE and then transferred to Hybond $^{\mathrm{TM}}$ polyvinylidene fluoride membranes (GE Healthcare 
Table I. Expression of $\beta$-catenin according to the clinicopathological factors of breast cancer.

\begin{tabular}{lcccc}
\hline & \multicolumn{3}{c}{$\beta$-catenin expression, $\mathrm{n}$} \\
\cline { 2 - 4 } & $\begin{array}{c}1+ \\
\text { Characteristics }\end{array}$ & $\begin{array}{c}2+ \\
(\mathrm{n}=14)\end{array}$ & $\begin{array}{c}3+ \\
(\mathrm{n}=32)\end{array}$ & P-value \\
\hline Tumor size & & & & 0.21 \\
T1 & 4 & 11 & 8 & \\
T2 & 8 & 15 & 21 & \\
T3 & 2 & 1 & 1 & \\
T4 & 0 & 1 & 2 & \\
Lymph node & & & & 0.04 \\
N0 & 9 & 11 & 13 & \\
N1 & 3 & 11 & 11 & \\
N2 & 1 & 4 & 7 & \\
N3 & 1 & 2 & 1 & \\
TNM stage & & & & \\
I & 5 & 9 & 7 & 0.03 \\
II & 4 & 13 & 17 & \\
III & 5 & 6 & 8 & \\
ER status & & & & \\
Negative & 0 & 13 & 11 & \\
Positive & 14 & 15 & 21 & \\
\hline
\end{tabular}

TNM, tumor-node-metastasis; ER, estrogen receptor.

Life Sciences, Chalfont, UK). Each membrane was blocked for $2 \mathrm{~h}$ with $5 \%$ skim milk and incubated overnight at $4^{\circ} \mathrm{C}$ with the $\beta$-catenin, $\beta$-actin, cyclin D1 or p-AKT antibody (all diluted at $1: 2,500$ in $5 \%$ skimmed milk/PBS buffer). HRP-conjugated $\operatorname{IgG}(1: 2,000$ dilution) was used as the secondary antibody for $1 \mathrm{~h}$ at $4^{\circ} \mathrm{C}$. Protein levels were determined using a Fujifilm Image Analyzer (Tokyo, Japan). Immunoreactive signal were visualized with western chemiluminescent HRP substrate (Merck KGaA). The $\beta$-catenin-relative density of the electrophoretic band was obtained with LAS-1000 Intelligent Dark-Box II (Fujifilm Corporation, Tokyo, Japan).

Statistical analysis. Statistical analysis was performed using SPSS version 15.0. (SPSS, Inc., Chicago, IL, USA). Statistical data analysis was performed using analysis of variance and Duncan's test. $\mathrm{P}<0.05$ was considered to indicate a statistically significant difference.

\section{Results}

Patients' characteristics. The present cohort comprised 74 female patients of ages ranged from 32 to 78 years (mean, 50.7 years), who were diagnosed with invasive ductal carcinoma and underwent curative surgery. In total, 44 (59.5\%) patients had T2 tumors on presentation, 23 (31.1\%) patients were T1, 4 (5.4\%) patients were T3 and $3(4.1 \%)$ patients were T4. Lymph node involvement status was N0 in $33(44.6 \%)$ patients, while $25(33.8 \%)$ patients were N1, 12 (16.2\%) were $\mathrm{N} 2$ and $4(5.4 \%)$ were N3. In total, 21 (28.4\%) patients were
$\mathbf{A}$
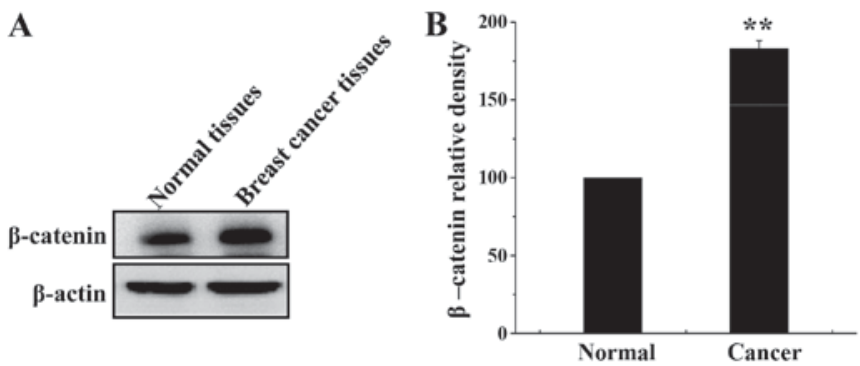

Figure 1. Expression profiles of $\beta$-catenin in homogenized breast cancer tissues. (A) $\beta$-catenin protein expression was analyzed by western blotting. $\beta$-actin was used as a loading control. (B) $\beta$-catenin relative to $\beta$-actin density. Values were normalized to the control (normal tissues). Error bars represent the standard deviation $(\mathrm{n}=74) .{ }^{* *} \mathrm{P}<0.005$ compared with the normal tissues.

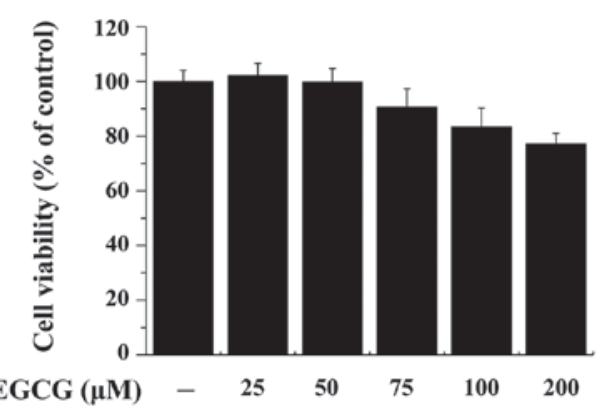

Figure 2. Effect of EGCG on the viability of MDA-MB-231 cells. MDA-MB-231 cells were treated with various concentrations of EGCG for $24 \mathrm{~h}$. MTT assay was used to detect the viability of the cells. The optical density value of the untreated cells was regarded as $100 \%$. Each value represents the mean \pm standard error of the mean of three independent experiments. EGCG, epigallocatechin gallate.

stage I, 34 (45.9\%) were stage II and 19 (25.7\%) were stage III. Among all patients, $50(67.6 \%)$ were ER positive and 24 (32.4\%) were ER negative.

Expression of $\beta$-catenin in breast cancer patients. $\beta$-catenin expression was analyzed in breast cancer and normal tissues by western blotting. $\beta$-catenin was overexpressed in breast cancer tissue compared with its expression in normal tissue (Fig. 1A and B).

Association between $\beta$-catenin expression and patients' clinicopathological features. $\beta$-catenin expression in tumor cells was scored as $3+$ (an intensity of $>2 / 3$ of the adjacent normal epithelium), $2+(1 / 3-2 / 3$ of the adjacent normal epithelium) and $1+(<1 / 3$ of the adjacent normal epithelium). In total, $14(18.9 \%)$ patients had $1+\beta$-catenin expression, 28 $(37.8 \%)$ had $2+$ expression and $32(43.2 \%)$ had $3+$ expression. $\beta$-catenin expression was associated with lymph node metastasis $(\mathrm{P}=0.04)$, TNM stage $(\mathrm{P}=0.03)$ and ER status $(\mathrm{P}<0.01)$ (Table I). Other factors were not associated with $\beta$-catenin expression.

Effect of EGCG on MDA-MB-231 cell viability. MDA-MB-231 cells were treated with EGCG $(0-200 \mu \mathrm{M})$ for $24 \mathrm{~h}$, and toxicity was analyzed using an MTT assay. EGCG decreased MDA-MB-231 cell viability in a dose-dependent manner (Fig. 2). 
A

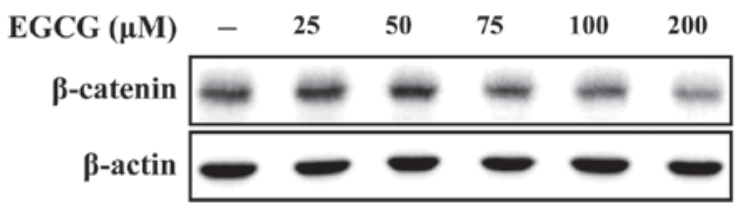

B

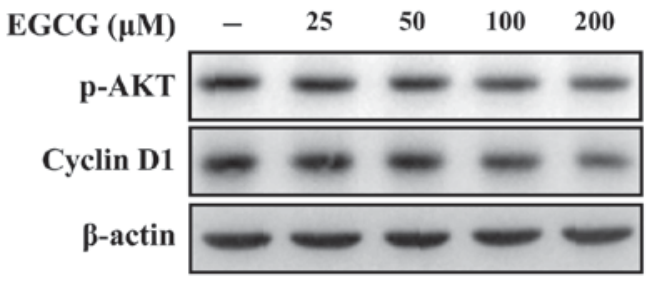

Figure 3. EGCG inhibits $\beta$-catenin, p-Akt and cyclin D1 expression in MDA-MB-231 cells. MDA-MB-231 cells were treated with EGCG for 24 h. (A) $\beta$-catenin protein expression was analyzed by western blotting. (B) p-Akt and cyclin D1 protein expression were analyzed by western blotting. $\beta$-actin was used as a loading control. Each value represents the mean \pm standard error of the mean of three independent experiments. EGCG, epigallocatechin gallate; p-, phosphorylated.

\section{A}

\begin{tabular}{|c|c|c|c|c|c|c|}
\hline EGCG $(200 \mu M)$ & - & + & - & - & + & + \\
\hline LY294002 ( $\mu \mathrm{M})$ & - & - & 25 & - & 25 & - \\
\hline Wortmannin $(\mu \mathrm{M})$ & - & - & - & 5 & - & 5 \\
\hline$\beta$-catenin & -8 & $=0$ & $=0$ & - & - & 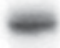 \\
\hline$\beta$-actin & & & & $\infty$ & & \\
\hline
\end{tabular}

B

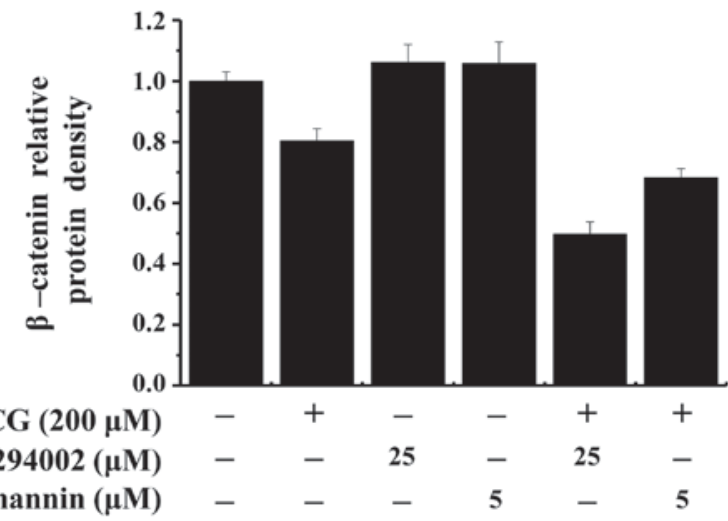

Figure 4. EGCG inhibits $\beta$-catenin through the PI3K signaling pathway in MDA-MB-231 cells. (A) MDA-MB-231 cells were pretreated with PI3K inhibitors (LY294002 and wortmanin), and then EGCG was added for $24 \mathrm{~h}$. b-catenin protein expression was analyzed by western blotting. $\beta$-actin was used as a loading control. (B) Quantification of $\beta$-catenin relative density. Each value represents the mean \pm standard error of the mean of three independent experiments. PI3K, phosphatidylinositol-3 kinase; EGCG, epigallocatechin gallate.

Effect of EGCG on $\beta$-catenin, phosphorylated (p)-Akt and cyclin D1 expression in MDA-MB-231 cells. Western blot analysis indicated that treatment of MDA-MB-231 cells with EGCG for $24 \mathrm{~h}$ suppressed $\beta$-catenin expression in a dose-dependent manner (Fig. 3A). To investigate the effect of EGCG on the phosphorylation of Akt and cyclin D1 activity, western blotting was performed. Treatment of MDA-MB-231 cells with EGCG for $24 \mathrm{~h}$ decreased p-Akt and cyclin D1 expression (Fig. 3B).

Effect of EGCG treatment with PI3 kinase inhibitors on $\beta$-catenin expression in MDA-MB-231 cells. PI3 kinase-dependent signaling pathway may be involved in the regulation of $\beta$-catenin expression, in combination with other signal transduction pathways. MDA-MB-231 cells were pretreated with PI3 kinase inhibitors for $1 \mathrm{~h}(25 \mu \mathrm{M}$ LY294002 or $5 \mu \mathrm{M}$ wortmannin), and EGCG was added for
24 h. EGCG with LY294002 or wortmannin exhibited an additive effect on $\beta$-catenin suppression in MDA-MB-231 cells (Fig. 4A and B). These results suggest that EGCG inhibited the growth of MDA-MB-231 cells through inactivation of the $\beta$-catenin signaling pathway.

\section{Discussion}

Breast cancer is the most frequently diagnosed cancer in women worldwide and the second most common cause of cancer mortality (23). Advances in cancer genomics have clarified the intrinsic subtypes of breast cancer, resulting in targeted treatment, including endocrine therapy and anti-HER2 therapy for patients with breast cancer who have HR- or HER2-positive tumors $(1,4)$. The success of target treatment has been integral to the survival improvement in patients with breast cancer (24). However, TNBC patients, who account for $15-20 \%$ of all breast cancer patients, have no target treatment and suffer shorter survival than patients with other subtypes of breast cancer (25). Additionally, TNBC is remarkably heterogeneous at the transcriptional level, and this is the main barrier for improving survival and developing target treatment for patients with TNBC (25). Thus, there are numerous studies on new drugs or combination treatments with conventional drugs for TNBC, including research into alternative treatments with natural herbal regimens such as polyphenols $(5,6)$. Furthermore, polyphenols induce restoration of tamoxifen sensitivity in MDA-MB-231 cells (7).

The Wnt signaling pathway is an important developmental pathway that is frequently dysregulated in human cancer (19). Wnt ligands primarily signal via membrane-bound Frizzled receptors through a number of different but interconnected signaling pathways, including the $\beta$-catenin, $\mathrm{Wnt} / \mathrm{Ca}^{2+}$ and planar-cell polarity signaling pathways (19). $\beta$-catenin is a transcriptional activator that activates target genes in the nucleus (25). Free cytoplasmic $\beta$-catenin may translocate to the nucleus and activate the Wnt signaling pathway, which contributes to tumorigenesis and progression in numerous organs, including the breast (25). Aberrant expression of $\beta$-catenin is associated with increased invasion, metastasis and poor prognosis in patients with breast cancer (26). Consistent with this, the present study revealed that $\beta$-catenin is overexpressed in breast cancer tissue compared with its expression in normal tissue, and that $\beta$-catenin expression is associated with lymph node metastasis, high TNM stage and ER-negative status.

Flavonoids are low-molecular weight, plant-derived compounds present in fruits, vegetables, herbs, tea and 
wine (20). They are divided into different classes, including polyphenols, which are particularly concentrated in green tea (Camellia sinensis), accounting for 30-40\% of its dry weight, while other flavonoids are present only in small quantities in green tea (15). EGCG, the most abundant polyphenol in green tea, is linked to the majority of health benefits associated with green tea consumption $(8,9)$. Green tea and its major constituent, EGCG, have been extensively studied as potential treatments for a variety of diseases, including cancer $(14,15)$. EGCG exerts its anticancer effects through multiple mechanisms, including anti-oxidation, induction of apoptosis, inhibition of angiogenesis and metastasis (10). Epidemiological data suggest that EGCG protects against hormone-associated cancers, including breast cancer (15). EGCG can prevent and inhibit breast tumorigenesis independently of the ER status (16), and is cytotoxic toward breast cancer cells regardless of their ER status. Following treatment with EGCG, cell numbers were significantly lower in ER-positive and ER-negative cell lines compared with those in the control $(7,18)$. In the present study, EGCG decreased MDA-MB-231 cell viability in a dose-dependent manner.

$\beta$-catenin is an important protein in the progression of multiple epithelial malignancies (20). A previous study treating MCF-7 cells with EGCG observed a reduction in $\beta$-catenin protein content and messenger RNA expression (13). In the present study, western blot analysis indicated that the protein levels of $\beta$-catenin in whole cell lysates of MDA-MB-231 cells were significantly reduced following incubation with EGCG for $24 \mathrm{~h}$ in a dose-dependent manner.

PI3 kinases serve key roles in cell proliferation, migration, apoptosis, gene expression and differentiation (27). Numerous proteins have been identified as direct or indirect downstream targets of PI3 kinase, and the most explored effector of the PI3 kinase signaling pathway is Akt (28). Akt, also known as protein kinase $\mathrm{B}$, is a serine/threonine kinase that is pivotal in cellular metabolism, growth and survival (28). The Akt signaling pathway is also a target for flavonoids such as EGCG (14). A previous report indicated that EGCG inhibits the PI3 kinase/Akt/mammalian target of rapamycin signaling pathway (29). The current study noticed that EGCG effectively inhibited p-Akt. Furthermore, the reduction in p-Akt by EGCG pretreatment was dose dependent. Cyclin D1 is a member of the cyclin protein family, and is involved in regulating cell cycle progression (14). The synthesis of cyclin D is initiated during G1, and drives the G1/S phase transition (14). Khan et al (14) reported that EGCG decreased the expression of cyclin D1. The present study also demonstrated that incubation with EGCG for $24 \mathrm{~h}$ reduced cyclin D1 expression in MDA-MB-231 cells.

Soluble $\beta$-catenin has the ability to bind PI3 kinase, which may mediate $\beta$-catenin stabilization (27). Thus, the inhibition of PI3 kinase may be responsible for the reduced expression of p-Akt and $\beta$-catenin observed in the present study. A previous study indicated that the PI3 kinase-dependent signaling pathway may be involved in the regulation of $\beta$-catenin expression, together with other signal transduction pathways (29). Therefore, the present study investigated the effect of PI3 kinase and EGCG on $\beta$-catenin expression, and the results revealed that inhibition of PI3 kinase with LY294002 and wortmannin displayed an additive effect with EGCG by considerably reducing the expression of $\beta$-catenin. These data may account for the anticancer effect of EGCG via $\beta$-catenin inhibition in MDA-MB-231 cells.

In conclusion, the present study provided associations between $\beta$-catenin expression and poor prognostic factors of breast cancer, and suggested that EGCG inactivates the $\beta$-catenin signaling pathway in MDA-MB 231 breast cancer cells. To the best our knowledge the current study is the first to demonstrate that EGCG suppresses cell proliferation and disrupts adherence junction formation via inhibition of the $\beta$-catenin signaling pathway in the MDA-MB 231 cell line. In summary, the present findings suggest that EGCG could be considered a potential treatment drug for TNBC patients.

\section{Acknowledgements}

The present study was supported by grants from the Biomedical Research Institute, Chonbuk National University Hospital in 2011 and a National Research Foundation (NRF) of Korea grant provided by the Korean government (grant no. NRF-2013R1A1A2011718).

\section{References}

1. Perou CM, Sørlie T, Eisen MB, van de Rijn M, Jeffrey SS, Rees CA, Pollack JR, Ross DT, Johnsen H, Akslen LA, et al: Molecular portraits of human breast tumours. Nature 406: 747-752, 2000

2. Ferlay J, Soerjomataram I, Dikshit R, Eser S, Mathers C, Rebelo M, Parkin DM, Forman D and Bray F: Cancer incidence and mortality worldwide: Sources, methods and major patterns in GLOBOCAN 2012. Int J Cancer 136: E359-E386, 2015.

3. Roodi N, Bailey LR, Kao WY, Verrier CS, Yee CJ, Dupont WD and Parl FF: Estrogen receptor gene analysis in estrogen receptor-positive and receptor-negative primary breast cancer. J Natl Cancer Inst 87: 446-451, 1995.

4. Gadducci A, Biglia N, Sismondi P and Genazzani AR: Breast cancer and sex steroids: Critical review of epidemiological, experimental and clinical investigations on etiopathogenesis, chemoprevention and endocrine treatment of breast cancer. Gynecol Endocrinol 20: 343-360, 2005.

5. Sartippour MR, Pietras R, Marquez-Garban DC, Chen HW, Heber D, Henning SM, Sartippour G, Zhang L, Lu M, Weinberg $\mathrm{O}$, et al: The combination of green tea and tamoxifen is effective against breast cancer. Carcinogenesis 27: 2424-2433, 2006.

6. Sak K: Chemotherapy and dietary phytochemical agents. Chemother Res Pract 2012: 282570, 2012.

7. Chisholm K, Bray BJ and Rosengren RJ: Tamoxifen and epigallocatechin gallate are synergistically cytotoxic to MDA-MB-231 human breast cancer cells. Anticancer Drugs 15: 889-897, 2004.

8. Graham HN: Green tea composition, consumption, and polyphenol chemistry. Prev Med 21: 334-350, 1992.

9. Nakachi K, Matsuyama S, Miyake S, Suganuma M and Imai K: Preventive effects of drinking green tea on cancer and cardiovascular disease: Epidemiological evidence for multiple targeting prevention. Biofactors 13: 49-54, 2000.

10. Kurahashi N, Sasazuki S, Iwasaki M, Inoue M and Tsugane S; JPHC Study Group: Green tea consumption and prostate cancer risk in Japanese men: A prospective study. Am J Epidemiol 167: 71-77, 2008.

11. Yang CS, Landau JM, Huang MT and Newmark HL: Inhibition of carcinogenesis by dietary polyphenolic compounds. Annu Rev Nutr 21: 381-406, 2001.

12. Zhang M, Holman CD, Huang JP and Xie X: Green tea and the prevention of breast cancer: A case-control study in Southeast China. Carcinogenesis 28: 1074-1078, 2007.

13. Hsu YC and Liou YM: The anticancer effects of (-)-Epigalocathine-3-gallate on the signaling pathways associated with membrane receptors in MCF-7 cells. J Cell Physiol 226: 2721-2730, 2011 
14. Khan N, Afaq F, Saleem M, Ahmad N and Mukhtar H: Targeting multiple signaling pathways by green tea polyphenol (-)-epigallocatechin-3-gallate. Cancer Res 66: 2500-2505, 2006.

15. Stuart EC, Scandlyn MJ and Rosengren RJ: Role of epigallocatechin gallate (EGCG) in the treatment of breast and prostate cancer. Life Sci 79: 2329-2336, 2006.

16. Thangapazham RL, Singh AK, Sharma A, Warren J, Gaddipati JP and Maheshwari RK: Green tea polyphenols and its constituent epigallocatechin gallate inhibits proliferation of human breast cancer cells in vitro and in vivo. Cancer Lett 245: 232-241, 2007.

17. Goodin MG, Fertuck KC, Zacharewski TR and Rosengren RJ Estrogen receptor-mediated actions of polyphenolic catechins in vivo and in vitro. Toxicol Sci 69: 354-361, 2002.

18. Roy AM, Baliga MS and Katiyar SK: Epigallocatechin-3-gallate induces apoptosis in estrogen receptor-negative human breast carcinoma cells via modulation in protein expression of p53 and Bax and caspase-3 activation. Mol Cancer Ther 4: 81-90, 2005.

19. Boras-Granic K and Wysolmerski JJ: Wnt signaling in breast organogenesis. Organogenesis 4: 116-122, .2008.

20. Amado NG, Fonseca BF, Cerqueira DM, Neto VM and Abreu JG: Flavonoids: Potential Wnt/beta-catenin signaling modulators in cancer. Life Sci 89: 545-554, 2011.

21. Loh YN, Hedditch EL, Baker LA, Jary E, Ward RL and Ford CE: The Wnt signaling pathway is upregulated in an in vitro mode of acquired tamoxifen resistant breast cancer. BMC Cancer 13 $174,2013$.
22. Edge SB, Byrd DR, Compton CC, Fritz AG, Greene FI and Trotti A III: Part VII breast. AJCC Cancer Staging Manual. 7th edition. Springer, New York, NY, pp347-376, 2010.

23. Parkin DM, Bray F, Ferlay J and Pisani P: Global cancer statistics, 2002. CA Cancer J Clin 55: 74-108, 2005.

24. Piccart-Gebhart MJ, Procter M, Leyland-Jones B, Goldhirsch A, Untch M, Smith I, Gianni L, Baselga J, Bell R, Jackisch C, et al: Trastuzumab after adjuvant chemotherapy in HER2-positive breast cancer. N Engl J Med 353: 1659-1672, 2005.

25. Foulkes WD, Smith IE and Reis-Filho JS: Triple-negative breast cancer. N Engl J Med 363: 1938-1948, 2010.

26. López-Knowles E, Zardawi SJ, McNeil CM, Millar EK, Crea P, Musgrove EA, Sutherland RL and O'Toole SA: Cytoplasmic localization of beta-catenin is a marker of poor outcome in breast cancer patients. Cancer Epidemiol Biomarkers Prev 19: 301-309, 2010.

27. Nelson CM and Chen CS: Cell-cell signaling by direct contact increases cell proliferation via a PI3K-dependent signal. FEBS Lett 514: 238-242, 2002.

28. Toker A and Newton AC: Cellular signaling: Pivoting around PDK-1. Cell 103: 185-188, 2000.

29. Peairs A, Dai R, Gan L, Shimp S, Rylander MN, Li L and Reilly CM: Epigallocatechin-3-gallate (EGCG) attenuates inflammation in MRL/lpr mouse mesangial cells. Cell Mol Immunol 7: 123-132, 2010 . 